ORIGINAL ARTICLE

\title{
EFFECTS OF INFORMATION DISSEMINATION USING VIDEO OF INDIGENOUS LANGUAGE ON 11-12 YEARS CHILDREN'S DENTAL HEALTH
}

\author{
Bankole Olubunmi ${ }^{1}$, Ibiyemi Olushola ${ }^{2}$
}

\begin{abstract}
BACKGROUND: Videos as a medium of health education are useful tools. This study evaluated the effectiveness of a dental health education video in the Yoruba language (spoken in southwestern Nigeria) targeted at children from the lower socioeconomic class.

METHODS: An interventional study was conducted among 120 children aged 11 and 12 years, randomly selected from three public primary schools in Ibadan, Nigeria. Participants were assigned into three study groups: group 1 watched the video, group 2 received verbal dental health education in the Yoruba language and group 3 were the control. Following this, each participant received a full mouth prophylaxis, and six weeks later, their oral hygiene was assessed using the Simplified Oral Hygiene Index of Greene and Vermillion.

RESULTS: A mean debris score of (1.11), (1.04) and (1.57) was recorded for the video, verbal and control groups respectively $(p<0.001)$. The mean calculus index score was lowest among the verbal group (0.56), followed by the video group (0.75) and highest among the control (1.16) (p<0.001). However, multivariate analysis, controlling for child's age and fathers education, revealed that oral hygiene of the participants in the video group was significantly better by $28.6 \%$ compared to the control group while in the verbal education group there was an improvement of $23.4 \%$ in contrast to the control .

CONCLUSION: This study demonstrated that a culturally appropriate video in an indigenous language can significantly improve oral hygiene among school children from the lower socioeconomic group in Nigeria.

KEYWORDS: Dental health education, Video, Effectiveness, Nigeria, Child
\end{abstract}

DOI: http://dx.doi.org/10.4314/ejhs.v23i3.2

\section{INTRODUCTION}

Studies have revealed a high prevalence of poor oral hygiene among children in developing countries, particularly among children from the lower socioeconomic class $(1,2)$. This trend is also observed in Nigeria (3-6). Reasons for this observation include less awareness about oral hygiene methods and inadequate access to cleaning agents (1).

Dental health education is an effective means of prevention of dental diseases in children especially when the implementation is supported with teaching aids. In Nigeria, oral health programmes have been carried out in schools, but most have teachings been delivered verbally with demonstrations and teaching aids.

Videos as a medium of dental health education have been employed in many countries and reports (7-8) have shown them to be invaluable visual aids. Also, previous reports (710) demonstrated high levels of effectiveness when such aids were used as health education tools among caregivers, children and the elderly.

Nigeria is a developing country in West Africa where about $70 \%$ of the population live

\footnotetext{
${ }^{1}$ Department of Oral Health, University of Ibadan, Oyo, Nigeria

2Department of Periodontology \& Community Dentistry, University of Ibadan, Oyo, Nigeria

Corresponding Author: Bankole Olubunmi, Email: bumbank2002@yahoo.com
} 
below poverty line (11) thus implying that the majority of the people belong to the lower social class. The Yuruba Tribe is a large ethno-linguistic group in West Africa and they constitute about $21 \%$ of Nigeria's population (12). Chronic periodontal diseases have been found to be highly prevalent among Nigerians for decades and studies have shown that over $75 \%$ of Nigerians need scaling and polishing and plaque control (13). Research on different population groups in Nigeria indicates a low awareness and poor attitude to oral health care regardless of education $(14,15)$. Failure to access oral health care in Nigeria has been found to be as a result of lack of perceived need for treatment by the populace. This is considered to be a function of the level of awareness of oral diseases and their prevention and it is thought that this gap may be bridged by oral health education (16).

It has been observed that in recent times, the home video trade has thrived in Nigeria and the populace especially the lower social class appear to have a greater preference for local films depicting their culture and tradition. Children too have been observed to spend much time watching for these videos either in their homes or in neighborhood. They are attracted more to these videos because they are cultural, colorful, and watched in a relaxed atmosphere. Therefore, a culturally appropriate health education video in the Yoruba language titled "Itoju Eyin" (meaning the care of the teeth) (17) was developed and targeted at children from the lower socioeconomic class since research findings in Nigeria has reported poor oral hygiene among them (3-6).

The purpose of the video was to improve oral hygiene in children by highlighting the causes of poor oral hygiene, demonstrating the proper method of tooth brushing and educating them about ways of preventing poor oral hygiene. However, the effectiveness of this video in improving oral hygiene of children has to be evaluated. Therefore, this study evaluated the effectiveness of the video as a dental health education tool among Yoruba speaking children in Nigeria.

\section{MATERIAL AND METHODS}

An interventional study to evaluate the effectiveness of a dental health education video in the Yoruba language, was carried out in Idikan, a suburban area in Ibadan South West Local Government Area of Ibadan. Idikan is located in the inner city of Ibadan, the capital of Oyo State in South Western Nigeria. It is predominantly a Yoruba speaking and low socioeconomic community (18).

A previous study (6) reported that the prevalence of poor oral hygiene among children in a rural Nigerian community was $13.6 \%$. A difference in proportion calculated as $37 \%$ with a power of 0.9 at a significant level of 0.05 was used to determine a minimum sample of 28 subjects per group. However, 40 participants were recruited to allow for attrition.

A list of all the public primary schools in Idikan area was obtained from the Oyo State Local Inspectorate of Education office and three schools were randomly selected from a list of five primary schools in the area. Further, randomization was done blindly by an independent person to assign the three school groups into the various interventions. The first ballot picked was labeled Group 1, the second Group 2 and the third Group 3. Intervention 1 (video health education) was applied to group 1, Intervention 2 (verbal health education) was applied to group 2 and the third group was used as a control. All pupils between the ages of 11 and 12 years were identified from their school register using their date of birth. In the selected schools, forty pupils were randomly selected by balloting in each school to participate in the study. Inclusion criteria were the child's age (11-12 years) and presence of all to be scored teeth in child's mouth. Exclusion criteria included children with designated teeth to be scored missing (determined by oral examination) or those who when asked claimed to have received dental prophylaxis in the last month prior to the study. None of the recruited children claimed to have visited the dentist. All the recruited participants met the inclusion criteria.

Prior to the commencement of the intervention study, permission was obtained from the school authorities and written informed consent in Yoruba language was obtained from parents or guardians. The study was carried out in strict compliance with the Helsinki Declaration of the 1975, as revised in 1983 involving human subjects in which no harm was caused and each of the participants were fairly treated. 
Pre-intervention evaluation was initially conducted using the Simplified Index of Green and Vermillion (15) to give an overall oral hygiene status of the participants in the three schools. The Debris Index Scores (DI-S) and Calculus index Scores (CI-S) was obtained for each patient and the Simplified Oral Hygiene Index (S-OHI) score for each individual was calculated as (DI-S + CI-S)/6. In applying the index, six designated teeth $16,11,26,46,31$ and 36 were scored. The buccal surfaces of 16, 26; the labial surfaces of 11,31 and the lingual surfaces were 46 , and 36 were scored. Plaque and calculus were graded on a numerical scale from 0 to 3 depending on the severity and extent of the deposits using the Debris Index Scores (DI-S) and Calculus Index Scores (CI-S).

Participants in intervention group 1 (the video education group) were invited to the school hall to watch the video titled "Itoju Eyin" (17)' They sat comfortably in front of a 40 inch television screen. The video lasted for 20 minutes and it was only watched once. The message in this video was delivered in an interesting and entertaining manner. The video was made up of a cast of local actors who perform in home videos often watched by these children. The video was filmed in a local traditional setting in the local language.

The story is about two contrasting families from low socioeconomic backgrounds. The first is Kunle's (a nine year old boy) family where there is neglect of oral hygiene resulting in very poor oral health. The second is Bola's (a nine year old girl) family in which healthy diet was consumed and the teeth were properly taken care of resulting in good oral hygiene.

Participants in intervention group 2 (verbal education group) received verbal dental health education which had the main message similar to that in the video "Itoju Eyin", in the Yoruba language The verbal dental health education was given once for a period of twenty minutes. The oral health educator who was a dentist used samples of cariogenic diets and pictures to demonstrate causes of poor oral health (tooth decay). The frequencies of tooth-brushing and correct tooth-brushing techniques were also demonstrated. However, the participants in the control group received no dental health education intervention.
Afterwards, each participant was given a full mouth prophylaxis, a new toothbrush and toothpaste of the same brand. All treatments were carried out at the Primary Oral Health Care Centre located in the local community by the six dental house officers who had a one day training, prior to the commencement of the study, on how scaling and polishing should be done. Immediately after the treatment, four independent examiners blinded to the study groups, examined the oral hygiene of the participants using the Simplified Oral Hygiene Index of Greene and Vermilion (19), to ensure they all had zero scores. Before, the examination, the examiners had a one day training on the application of the index.

Six weeks after treatment, post-intervention evaluation of the participants was conducted by the same independent evaluators using the Simplified Oral Hygiene Index of Greene and Vermilion. At each post-treatment evaluation, duplicate examinations were carried out on a random sample of $10 \%$ of the subjects. The intra and inter examiner reliability was calculated from the percentage agreements and was found to be greater than $90 \%$.

All follow-up examinations were done in schools in order to minimize loss of subjects, and a careful record of each participant was documented. There was a schedule that ensured effective monitoring and follow-up of the participants. There was no subject lost to followup in this study as the students were all present in school during post-intervention evaluation.

Data were entered and analyzed using Statistical Package for Social Science (SPSS) version 17. Frequencies, proportions and means were generated; t-test and ANOVA were used to compare means at $5 \%$ level of significance. Multiple logistic regressions with a multinomial approach were used to determine the effectiveness of the intervention with adjustments for the child's age and father's education.

\section{RESULTS}

A total of 120 pupils aged 11-12 years with a mean age of $11.5+0.78$ years, assigned in three groups, participated in the study. Group 1 consisted of 22 males and 18 females, and Group 2, 21 females and 19 males. In group 3, there were 18 male and 22 female children. 
Table 1 reveals that the mean debris index score was lowest (1.04) among the group that received health education verbally while it was the highest among the control group (1.57). A mean debris score of (1.11) was recorded among the group that received health education by watching the video. These differences in the mean debris score among the three groups were statistically significant $(\mathrm{p}<0.001)$.

Table 1: Debris Index scores of 11-12 years old children in three primary schools in Idikan, Ibadan, December 2011

\begin{tabular}{lcccc}
\hline Debris Index Scores & & & & \\
Study Group & Mean & SD & F value & p value \\
\hline \multicolumn{5}{l}{ Pre-intervention } \\
Video & 1.88 & 0.65 & & \\
Verbal & 2.18 & 0.69 & & \\
Control & 2.02 & 0.55 & & \\
& Post intervention & 12.59 & 0.000 \\
Video & 1.11 & 0.34 & & \\
Verbal & 1.04 & 0.60 & & \\
Control & 1.57 & 0.56 & \\
\hline
\end{tabular}

There was a statistically significant difference in the mean calculus index scores among the three groups $(\mathrm{p}<0.001)$ (Table 2$)$. Those who received oral health education verbally had a mean calculus score of 0.56 ; those who had it by watching the video had a score of 0.75 , and those who did not receive health education had a score of 1.16 .

Table 2: Calculus Index scores of 11-12 years old children in three primary schools, Idikan, Ibadan, December 2011

\begin{tabular}{lcccc}
\hline \multicolumn{5}{l}{ Calculus Index Scores } \\
Study Group & Mean & SD & F value & p value \\
\hline \multicolumn{5}{c}{ Pre-intervention } \\
Video & 1.48 & 0.50 & & \\
Verbal & 1.64 & 0.47 & & \\
Control & 1.83 & 0.64 & & \\
& Post intervention & & \\
Video & 0.75 & 0.35 & & \\
Verbal & 0.56 & 0.36 & 22.42 & 0.000 \\
Control & 1.16 & 0.50 & & \\
\hline
\end{tabular}

Post-intervention Simplified Oral Hygiene Index scores were $0.93,0.80$, and 1.36 respectively for the video, verbal and control groups $(\mathrm{p}<0.001)$ Table 3. 
Table 3: Simplified Oral Hygiene Index scores of 11-12 year old children in three primary schools in Idikan, Ibadan in December 2011.

\begin{tabular}{|c|c|c|c|c|}
\hline \multicolumn{5}{|c|}{ Simplified Oral Hygiene Index Scores } \\
\hline Study Group & Men & $\mathrm{SD}$ & F value & $p$ value \\
\hline \multicolumn{5}{|c|}{ Pre-intervention } \\
\hline Video & 1.68 & 0.53 & & \\
\hline Verbal & 1.91 & 0.52 & & \\
\hline Control & 1.92 & 0.58 & & \\
\hline \multicolumn{5}{|c|}{ Post-intervention } \\
\hline Video & 0.93 & 0.30 & 18.65 & 0.000 \\
\hline Verbal & 0.80 & 0.46 & & \\
\hline Control & 1.36 & 0.51 & & \\
\hline
\end{tabular}

According to table 4, using the multivariate model, controlled for age and fathers education, results showed that in the group which watched the video, a decrease in the Oral Hygiene Index reduces the odd of the pupils hygiene by $28.6 \%$ when compared with the control group $(\mathrm{CI}=$ $(14.1 \%-57.9 \%), \mathrm{p}=0.001)$. The odd of the pupils who were exposed to verbal instruction was lower by $23.4 \%$ compared to the control group $(\mathrm{CI}=$ (11.9- 45.8), $\mathrm{p}=0.000$ ). Thus, oral hygiene of those exposed to the video was better by $28.6 \%$ compared to that of the control group. The oral hygiene of the students who received verbal education was improved by $23.4 \%$ in contrast to that of the control.

Table 4: Multiple logistic regressions with a multinomial approach analysis of the Simplified Oral Hygiene Index scores of 11-12 year old children in three primary schools in Idikan, Ibadan, December 2011.

\begin{tabular}{|c|c|c|c|c|c|}
\hline Variable & Coefficient B & SE(B) & P-value & Odds ratio & $\begin{array}{l}\text { 95\% CI for } \\
\text { OR }\end{array}$ \\
\hline \multicolumn{6}{|l|}{ Video } \\
\hline $\begin{array}{l}\text { OHI } \\
\text { Constant }\end{array}$ & $\begin{array}{l}-1.253 \\
-26.558\end{array}$ & $\begin{array}{l}0.360 \\
8.822\end{array}$ & 0.001 & 0.286 & $0.141,0.579$ \\
\hline \multicolumn{6}{|l|}{ Verbal } \\
\hline $\begin{array}{l}\text { OHI } \\
\text { Constant }\end{array}$ & $\begin{array}{l}-1.452 \\
-6.379\end{array}$ & $\begin{array}{l}0.343 \\
4.234\end{array}$ & 0.000 & 0.234 & $0.119,0.458$ \\
\hline
\end{tabular}

Adjusted for child's age and father's education

\section{DISCUSSION}

Oral health education, an important part of oral health promotion, has been considered an essential and basic part of oral health service especially when delivered verbally and with demonstrations through posters. It can also be delivered using videotapes in clinics, homes, theatres and schools. Various methods of delivering oral health messages can promote oral health by providing information that will improve awareness leading to adoption of healthier lifestyles, positive attitudes and good oral health behaviors (20). They are important for the development of good oral health in children since unhealthy habits and practices are acquired during childhood. It is widely understood that good oral health practices are necessary from a young age to ensure positive long term results (21).

In Nigeria, oral health education is usually delivered to children verbally and with demonstrations with posters in schools and clinics. However, there is a dearth of information on the effectiveness of oral health education delivered verbally to children in a local Nigerian language. There is also no documented report on the use of videotapes in oral health education among children in a local Nigerian language.

In this study, simplified oral hygiene index scores after a six week period in the video group was 
significantly better by $28.6 \%$ compared to the control group thus revealing effectiveness of the videotape. Oral hygiene in those who received verbal education improved by $23.4 \%$ in contrast to the control. Similarly, improvements in oral hygiene were demonstrated with the use of videos in a study comparing written, verbal and videotape oral hygiene instructions for patients with fixed appliances in studies by Lees and Rock (22) and Yazdani et al., which assessed improvement in oral cleanliness and gingival health among adolescents (23).

A remarkable feature about the video "Itoju eyin" was that it is culturally appropriate and was presented in an entertaining way. The cast consisted entirely of artistes who appear in the local home videos often watched by these children. It has been proven that people are usually more captivated with health promotion messages grounded in a story set in their own social and cultural context (24). The oral hygiene scores in the group taught verbally was significantly better by $23.4 \%$. This reveals that the verbal mode of oral hygiene instruction was effective. This may be attributed to the fact that the verbal session was interactive and the children could ask questions.

The children in this study watched the video only once and did not have repeated exposures unlike those in the study by Lees and Rock (22) who took the videos home. Repeated exposures may have further resulted in improvement of oral hygiene of the children in our study.

Videos in the local language spoken by the children will obviously increase understanding of the local health message. They can be vivid and graphic, providing the opportunity to act out reallife situations, demonstrate appropriate behavior, or simply present information in a more lively way (25). Videos can also provide the viewer with the opportunity of learning in privacy and comfort (26) and it can be watched repeatedly as often as it is required (27-28).

However, a major constraint in the use of the video in the rural and suburban Nigeria areas is one of power supply which is irregular. Power generating plants can; however, be acquired by schools, clinics for children to view this video. Furthermore, town halls in the rural and suburban areas are sometimes powered by generators to allow the local communities to watch football matches. Such centers can serve as good venues where this video can be shown to children from the lower social class.

This study demonstrated that a culturally appropriate video can significantly improve oral hygiene among school children from the lower socioeconomic group in Ibadan, Nigeria. Health education delivered verbally was also found effective. The videotape is effective and acceptable by the children. It is a potentially costeffective mode for health education and should be developed in Igbo language commonly spoken in eastern Nigeria and the Hausa language which is predominant in Northern Nigeria.

Furthermore, because of the fact that videos tape in an indigenous language has been found effective, health agencies in developing countries particularly in Africa can employ such entertaining culturally appropriate tools to enhance improved oral hygiene among children from the lower social class.

The videotape has been found to be effective, likewise the verbal mode of health education in children. It is recommended that the video be shown to children particularly those from the lower social group. However, it may be more effective if combined with other forms of education (e.g verbal education) especially in a country like Nigeria where electricity supply can be erratic.

LIMITATION OF THE STUDY: A larger sample size could have been better. There was limitation in the number of participants recruited due to the limited amount of funds. Each child's treatment had to be paid for at the primary health care centre amongst other expenses. Also, the participants in the video education group watched the videotape only once. Repeated exposures may have improved their oral hygiene scores. Variables such as effects of the presence of a primary oral care centre or having received previous oral health education information from other sources were not controlled and could have contributed to the heterogeneity within the study participants thus affecting the findings of this study.

This research was funded by the Senate Research Grant, University of Ibadan, Ibadan, Nigeria 


\section{REFERENCES}

1. Addo-Yobo C, Williams SA, Curzon ME. Oral hygiene practices, oral cleanliness and periodontal treatment needs in 12 year old urban and rural children in Ghana. Community Dental Health 1991; 8: 155-162.

2. Frenken JE, Truin GJ, van't Hoff M A, Konig K G, Lembariti BS, Mulder J, Kalsbeek H. Plaque ,calculus, gingival bleeding and type of tooth cleaning devise in a Tanzanian child population in 1984,1986,1988. Journal of Clinical Periodontology 1991; 8: 592-597.

3. Noah MO. Caries experience and state of oral cleanliness in 4 year old Ibadan children attending private schools. Journal of the International Association of Dentistry for Children 1981; 12: 17-23.

4. Aderinokun GA, Lawoyin JO, Onyeaso CO. Effect of two common Nigerian chewing sticks on gingival health and oral hygiene. Odontostomatologie tropicale 1999; 22:13-18.

5. Sofola OO, Shabar OP, Jeboda SO. Oral hygiene and periodontal treatment needs of urban school children compared with those of rural school children in Nigeria. African Dental Journal 1994; 8: 20-25.

6. Okolo SN, Chukwu GA, Egbuonu I, Ezeogu FA, Onwuanaku C, Adeleke OA, Hassan A, and Ngoe-Nesoah A. Oral Hygiene and Nutritional Status of Children Aged 1-7 Years in a Rural Community. Ghana Medical Journal 2006;40 : 22-25.

7. Laiho M. Honkala E, Nyyssonen V, Milen A. Three methods of oral health in secondary schools. Scandinavian Journal of Dental Research 1993;101: 442-447.

8. Paulin-Palokas T, Nordblad A, Remes-Lyly T. Video as a medium of oral health education for children with mental handicaps. Special Care in Dentistry 1997;17: 211-214.

9. Alsada LH, Sigal M J, Limback H, Fiege J, Kulkarni GV. Development and testing of an audiovisual aid for improving infant oral health through primary care giver education. Journal Canadian Dental Association 2005;71:241a241h.

10. Chalmers JM, Robinson J, Nankivell N. The practical oral care video evaluation of a dental awareness month initiative. Australian Dental Journal 2005;50:75-80.
11. Nigerian Poverty Profile 2010. Nigeria National Bureau of Statistics Available at www.nigerianstat.gov.ng

12. CIA. The World Factbook. Available at (www.cia.gov/library/publications/the-worldfactbook/geos/ni.html)

13. Sheiham A, Jeboda SO. Periodontal disease in Nigeria-The problem and possible solutions. Tropical Dental Journal 1981;4 (4): 211-219.

14. Agbelusi GA, Sofola OO, Jeboda SO. Oral health knowledge, attitude and practices of pregnant women in the Lagos University Teaching Hospital. Nigerian Quarterly Journal of Hospital Medicine. 1999; 9: 116-120.

15. Sofola OO, Agbelusi GA, Jeboda SO. Oral health knowledge, attitudes and practices of primary school teachers in Lagos State. Nigerian Medical Journal 2002; 11: 73-76.

16. Sofola OO. Implications of low oral health awareness in Nigeria. Nigerian Medical Journal 2010;15:131-133.

17. Bankole OO, Ibiyemi O, Oke GA. Developing a dental health education video for Nigerian children in the Yoruba language. African Journal of Biomedical Research 2011;14: 7779.

18. Aderinokun GA, Lawoyin JO, Faseemo VO, Bamgboye SM. Prevalence of tetracycline stained teeth among some school children in Ibadan, Nigeria. Nigerian Medical Practitioner 1994; 27:73-75.

19. Green JC and Vermillion JR. The Simplified Oral hygiene Index. Journal of American Dental Association 1964;68: 7-13.

20. Watt RG, Marinho VC. Does oral health promotion improve oral hygiene and gingival health? Periodontology 2000. 2005; 37:35-47.

21. Department of Health and Children(1999). Oral Health in Ireland. Oral Health Services Research Centre and the Irish Dental Health Foundation Dublin. In: Friel S, Hope A, Kelleher C, Comer $\mathrm{S}$, Sadlier D. Impact evaluation of an oral health intervention among primary school children in Ireland. Health Promotion International 2002; 17:120-126.

22. Lees A, Rock W. A comparison between written, verbal and videotape oral hygiene instruction for patients with fixed appliances. Journal of Orthodontics 2000; 27: 323-327.

23. Yazdani R, Vehkalahti MM, Nouri $M$, Murtomaa H. School-based education to improve oral cleanliness and gingival health in 
adolescents in Tehran, Iran. International Journal of Paediatric Dentistry 2009; 19:274281.

24. Mathews C, Guttmacher SJ, Coetzee N, Magwaza S, Stein J, Lombard C, Goldstein S, Coetzee D. Evaluation of a video based health education strategy to improve sexually transmitted disease partner notification in South Africa. Sexually Transmitted Infections 2002; 78:53-57.

25. Health Development Agency NHS. Is video an effective tool for health education? Available at www.nice.org.uk/niceMedia/documents/effective nessvideo.pdf pg 1.

26. Nielsen E and Sheppard M. Television as patient education tool. A review of its effectiveness. Patient Education and Counseling 1998;11: 216.

27. Spencer, K. Modes, Media and Methods. The Search for Educational Effectiveness, British Journal of Educational Technology 1991; 22: 12-22.

28. Ahmed, N. The use of Video Technology in Education. Educational Media International 1990; 27:119-123. 